\title{
Two Papers on Internet Connectivity and Quality
}

\author{
ROBERTO ROSON ${ }^{\circ}$ \\ Dipartimento di Scienze Economiche, \\ Università Ca'Foscari di Venezia, Venice, Italy.
}

\begin{abstract}
I review two papers, addressing the issue of competition and quality determination in the market for Internet access services (Crémer, Rey, and Tirole (2000), Foros and Hansen (2001)). These two works come to seemingly opposite conclusions. After having summarized the two papers, I try to highlight what lies behind the differences in the results.

Keywords: Internet, interconnection, quality, interface.
\end{abstract}

\section{$1 \quad$ Introduction}

Two papers have recently addressed the issue of competition and quality determination in the market for Internet access services. The first paper is by Crémer, Rey, and Tirole, published in the Journal of Industrial Economics (2000). The second paper is by Foros and Hansen, published in Information Economics and Policy (2001).

Interestingly, these two works come to seemingly opposite conclusions: whereas the first authors find that the bigger operators may want to degrade the quality of interconnections between networks (e.g., by lowering the capacity of interfaces), the second authors suggest that vertically differentiated firms, of different size, do not have conflicting interests with regard to interconnection quality.

The two models adopt different assumptions regarding, in particular, the effect of quality on the market dimension, the degree of firms' differentiation, and the nature

Cannaregio, S.Giobbe 873, Venezia, Italy. Tel. +39-041-2349164. E-mail: roson@unive.it 
of strategic instruments. Nonetheless, their findings are comparable, especially if vertical differentiation in the Foros-Hansen model is interpreted as the effect of the size of an installed base of customers.

In this review, I provide a summary of these two works and then I try to make a synthesis of the different positions, with the aim of highlighting what lies behind the differences in the results. I claim that the difference is driven by alternative hypotheses on the overall market size, which is variable in first paper but fixed in the second one.

Since Internet is an expanding business, the first approach seems to be more realistic. Nonetheless, it fails to address the heterogeneity of network services and the dynamic implications of the growing number of Internet subscribers.

\section{Paper \#1: Connectivity in the Commercial Internet}

The first paper deals with the "backbone" market in the Internet. Backbones constitute the top layer of the Internet hierarchical structure: users send/receive data to/from service providers (ISPs), then the providers communicate between themselves mainly through high-speed, high-capacity backbone connections.

Backbones are traditionally operated on the basis of "bill and keep" schemes: they reciprocally accept to route all traffic that is destined to their own customers (exchanged through public or private interfaces), and there are no charges applied between backbone operators.

Crémer, Rey, and Tirole stress, “connectivity requires cooperation among firms that are otherwise competitors. They must reach bilateral agreements on the locations and capacities of interfaces, and on the financial terms through which they exchange traffic". The paper focuses on the determinants of these agreements, highlighting the role played by the installed base of customers. To this end, the authors adopt and adapt the well-known model by Katz and Shapiro (1985).

The simplest version of the model considers a duopoly, in which the quality of service of backbone $i$, affecting the users willingness to pay ${ }^{1}$, is specified as:

$$
s_{i}=v\left[\left(\beta_{i}+q_{i}\right)+\theta\left(\beta_{j}+q_{j}\right)\right]
$$

\footnotetext{
${ }^{1}$ Consumer surplus is defined as $C S=T+s_{i}-p_{i}$, where the parameter $T$ is uniformly distributed in the population, giving raise to an aggregate linear demand curve.
} 
where $v$ is a valuation parameter, the $\beta$ s refer to the number of pre-existing users, and the $q$ s stand for the number of new users, subscribing either to backbone $i$ or to $j$. $\theta$ is a parameter, reflecting the quality of interconnection. It is comprised between zero and one: if it is zero, users of the backbone $j$ are unreachable by $i$-users; if it is one, there are no qualitative differences between inter- and intra-backbone connections.

The duopolists play the following game: first, they independently choose a value for the parameter $\theta$, and the lower value determines the actual interconnection quality; second, they set quantities (à la Cournot) for backbone services, so that new users choose where to subscribe, with no a priori preferences (in equilibrium, the choice is driven by an indifference condition, if both $q$ s are strictly positive).

Some remarks are in order here. Old users are captive and the competition is for attracting new subscribers. The authors justify this choice with the existence of large switching costs. However, if the subscription is a long term engagement, the users choice would be better specified in an intertemporal context. Indeed, the authors shows that, when connectivity is not perfect, the largest backbone becomes dominant in the market for new users. This would mean that the large operator becomes larger over time. Non-myopic users may anticipate all this and subscribe immediately to the main operator.

The authors demonstrate that the largest backbone chooses a lower interconnection quality, since this is a decreasing function of the difference in size of the installed bases. This result is due to two effects. First, a better interconnection quality raises the users' valuation of both backbones (because of network externalities, there is a demand expansion effect). Second, the two networks become more similar when connectivity is improved (the small network gains more in terms of access $^{2}$, that is, a differentiation effect). Since the two mechanisms influence the largest operator's profits in opposite ways, whereas they are both positive for the small backbone, the former chooses a lower interconnection quality (thereby determining the actual quality).

This insight is confirmed in a series of variant of the basic model in which: (1) quality is chosen cooperatively in a Nash bargain with side transfers, (2) the operators

\footnotetext{
${ }^{2}$ For example, suppose that one network has 50 subscribers and another one 100. If they choose to interconnect (perfectly), the smaller network can offer access to 150 subscribers $(+200 \%$ from its own base), whereas the bigger network can expand its access potential by only $50 \%$.
} 
play Bertrand in last stage of the game, (3) quality is endogenously determined on the basis of a chosen interface capacity, giving rise to traffic congestion.

The authors then turn to a more elaborated version of the model, considering four competitors that are initially assumed to have equal market shares. Because of this initial symmetry, none of the competitors would have incentives to set a poor interconnection quality. A merger between two operators is subsequently considered. The question then becomes: does the merger induce the largest operator to act anticompetitively, through a deterioration of its interconnection quality?

Observe that, by lowering quality in one interface, a large operator could benefit from a differentiation effect, but at the same time both interconnected backbones would loose demand vis-à-vis all other operators.

The authors claim that the big backbone would never choose a "global deterioration strategy", that is, lowering quality in all its interfaces. Instead, a "targeted degradation strategy", that is, directed towards only one of the smaller competitors, is said to be profitable: "If the other backbone does not provide any transit services," (and the paper discusses at length why this may indeed be the case) "the degradation strategy creates an asymmetry between the dominant backbone (who can offer access to three-fourth of the installed base) and the target (who can offer access to only half of the installed base).

\section{Paper \#2: Competition and Compatibility among Internet Service Providers}

The second paper consider a situation very similar to the duopoly model in the first paper, although the authors make no explicit reference to the backbone infrastructure, but only to some generic "service providers" (ISPs), giving access to the Internet.

Here, however, services are both vertically and horizontally differentiated. Following a conventional Hotelling framework, two competitors are virtually located at the two extremes of a segment, along which consumers with unitary demand are uniformously distributed. The utility obtained by a consumer, located in $x$, and subscribing to an ISP $i$, located in $x_{i}$, is defined as $^{3}$ :

\footnotetext{
${ }^{3}$ I have changed the notation here, to make it homogeneous with the notation adopted in (1).
} 


$$
U_{i}=w_{i}+t\left|x-x_{i}\right|+v\left(q_{i}+\theta q_{j}\right)-p_{i}
$$

where: $w$ is an "intrinsic" quality parameter, $t$ is the Hotelling transport factor, $v$ is a valuation parameter, $q$ stands for the number of subscribers of network $i$ or $j$, $\theta$ is the parameter of interconnection quality, as in (1), and $p_{i}$ is the subscription fee. As usual, each consumer chooses the network associated with the highest utility level. Parameters values are set in such a way that all consumers subscribe to one of the two ISPs. It is also assumed that there exists a consumer located at the boundary of the two market areas, which is indifferent about subscribing one network or the other (the authors focus only on interior solutions).

A game is played by the two firms. First, they cooperatively choose the level of the quality parameter in the range $0-1(0=$ disconnected networks, $1=$ fully integrated networks). Second, they autonomously set (à la Bertrand) the subscription fees.

Solving backwards, the market share of the ISP $i$ is found to be:

$$
\begin{aligned}
& \frac{q_{i}}{q_{i}+q_{j}}=\frac{1}{2}+\sigma\left(w_{i}-w_{j}\right)-\sigma\left(p_{i}-p_{j}\right) \\
& \sigma=1 /(2 t-2 v(1-\theta))
\end{aligned}
$$

Therefore, a better interconnection quality (or a lower sensitivity to network externalities) turns out to be equivalent to a higher degree of product differentiation. This implies that the two operators will choose the highest interconnection quality if this gives raise to negligible investment costs.

More surprisingly, the marginal profit associated to a change in the quality is equal for the two firms, which therefore always agree when choosing the characteristics of the network interface. This result comes from the overlapping of two effects: a price effect, which is positive for both ISPs (because a higher quality raises the consumers' valuation), and a market share effect, which has opposite sign for the two operators. In particular, the market share of the smaller network increases and the market share of the bigger network decreases. The first effect is dominant and sufficiently larger, for the bigger operator, to ensure the equality in the marginal profits.

When the costs of interconnection quality are increasing and convex, the quality level is found by equating marginal profits and marginal investment costs. The level chosen by the two competitors can then be compared with the socially optimal 
one, obtained by maximizing customer gross surplus minus total production cost.

In this model the quality level chosen by the two firms exceeds the socially optimal one. This is due to a strategic externality. Each firm raises its price twice: because of higher consumers' valuations, and because of the other competitor's price increase (prices are strategic complements).

\section{$4 \quad$ A Synthesis}

Different results are driven by different hypotheses, but not all differences are directly responsible for dissimilar (qualitative) findings.

Some diversity in basic assumptions is more superficial than substantial. For example, Crémer, Rey and Tirole consider only competition for new customers, whereas - in principle - customers may switch network in the Foros and Hansen paper. However, it is sufficient to reinterpret the quality differential $w$ in the latter paper as the valuation of the installed base (locked-in), and the consumers distributed in the Hotelling segment as new subscribers, to make the two models directly comparable in this respect.

Another obvious difference regards the nature of instruments (quantity vs. price) used in the second stage of the game, after the selection of the interconnection quality. It may be useful to think about the impact of an improved quality on profits as coming from two sources: the higher customers' valuation, and the change in the strategic behavior of the competitor. This secondary effect is different in the two cases, because quantities are strategic substitutes, whereas prices are strategic complements.

Still, this is not the primary determinant of the different outcomes, since in both cases the competitor who get a differential advantage gains a larger market share. The key element is the market expansion effect, triggered by a quality enhancement: this is present in the first paper, where an aggregate demand function (sensitive to quality) is used, but absent in the second paper, where the total number of subscribers is constant.

To see this, consider a simple variant of the Hotelling duopoly, in which the two markets are detached and there may be consumers not subscribing to either 
network ${ }^{4}$. When quality is improved, new customers may be captured by the two ISPs, so each individual market expands. However, since the smaller network always gains relatively more, in terms of access to network clients, it has also more incentives to improve the interconnection quality. When investment costs are convex, the bigger network would then prefer a lower quality level, as suggested by Crémer, Rey and Tirole.

Since the number of subscribers to Internet services is steadily increasing around the world, and their decision about if and where to subscribe may well be influenced by price and quality levels, the approach followed in the first paper seems closer to reality. However, Crémer, Rey and Tirole obtain their results in a static framework, which may not be appropriate to capture all the implications of a market whose size is growing over time.

Furthermore, vertical and horizontal differentiation (addressed only in the Foros and Hansen paper) is an important issue in the Internet. On one hand, geographical proximity influence the network choice, especially outside North America, and, on the other hand, backbone services are differentiated in several aspects (Gorman and Malecki (2000)).

If a variable market dimension is introduced in the Foros-Hansen model, it may not be true that the operators have equal incentives, with regard to the quality of interconnection. However, when competition is based on price setting, the insight that a better interconnection softens the competitive pressure is still valid, possibly bringing about a "too high" interconnection quality.

In any case, nothing ensures that the network operators autonomously select the right quality level, from a social point of view. In principle, then, there may be scope for regulatory intervention, whose analysis is however outside the scope of this paper. Yet, intervention would be necessary only in the presence of significant asymmetries between firms. In these circumstances, however, the network providers would probably prefer to abandon the "bill and keep" scheme, and transform it into a "transit" contract, in which the smaller network pays the bigger one for access services. Indeed, many big backbone operators already require that potential partners in peering agreements must satisfy certain requirements, in terms of: number and

\footnotetext{
${ }^{4}$ Alternatively, consumers are located also to the right and to the left of the two operators.
} 
characteristics of customers, number and location of interfaces, link capacity, etc.

\section{$5 \quad$ References}

Crèmer, J., Rey, P., Tirole, J. (2000), "Connectivity in the Commercial Internet", Journal of Industrial Economics, 48:433-472.

Foros, Ø, Hansen, J. (2001), "Competition and compatibility among Internet Service Providers", Information Economics and Policy, 13: 411-425.

Gorman, S. P., Malecki, E. J. (2000), "The networks of the Internet: an analysis of provider networks in the USA", Telecommunications Policy, 24 (2): 113-134.

Katz, M.L., Shapiro, C. (1985), "Network Externalities, Competition, and Compatibility", The American Economic Review, 75: 424-440. 